\title{
Avaliação da progênie de ovelhas Merino Australiano cruzadas com carneiro Dorper ou Southdown
}

\author{
[Evaluation of the progeny of Australian Merino ewes crossed \\ with Dorper or Southdown ram] \\ G. Bianchi ${ }^{1}$, S. Carvalho ${ }^{2,3}$, J. Rivero ${ }^{1}$ \\ ${ }^{1}$ Universidad de la República. Facultad de Agronomía. Estación Experimental \\ "Dr. Mario A. Cassinoni”. Ruta 3, km 363.500. Paysandú. Uruguay \\ ${ }^{2}$ Programa de pós-graduação - Universidade Federal de Santa Maria - Santa Maria, RS \\ ${ }^{3}$ Bolsista de pós-doutorado do CNPq
}

\begin{abstract}
RESUMO
O presente estudo objetivou avaliar o efeito do genótipo do carneiro (Dorper ou Southdown), do sexo e do tipo de parto sobre características de crescimento de 268 cordeiros filhos de ovelhas Merino Australiano. Até o desmame, todos os animais permaneceram sobre pastagem de trevo-vermelho (Trifolium pratense), chicória (Cychorium intibus), trevo-branco (Trifolium repens), festuca (Festuca arundinacea) e azevém (Lolium multiflorum), com carga animal de 10 ovelhas com seus cordeiros/ha. A partir do desmame $(29,95 \pm 4,94 \mathrm{~kg}$ de peso vivo e 109,45 \pm 7,41 dias de idade), os cordeiros permaneceram sobre a mesma pastagem utilizada durante a lactação, sendo terminados posteriormente sobre um cultivo de soja (Glycine max), com carga animal de 30 cordeiros/ha. Não foi verificado efeito do genótipo do carneiro $(\mathrm{P}>0,05)$ sobre nenhuma das variáveis analisadas. Por outro lado, do nascimento ao desmame e do desmame até o embarque dos cordeiros para abate, os machos (criptorquidas) apresentaram ganhos de peso diário superiores (241 e 212g/dia; 121 e 94g/dia, para machos e fêmeas, respectivamente; $\mathrm{P} \leq 0,001$ ), o que proporcionou um peso vivo ao embarque $14,9 \%$ superior em relação às fêmeas $(40,89$ e $35,59 \mathrm{~kg}$, para machos e fêmeas, respectivamente; $\mathrm{P} \leq 0,001)$. Os cordeiros de parto simples apresentaram maiores $(\mathrm{P} \leq 0,001)$ pesos vivos e medidas corporais ao nascimento, bem como maior velocidade de crescimento até o desmame (239 e $214 \mathrm{~g} /$ dia, parto simples e duplo, respectivamente; $\mathrm{P} \leq 0,001)$. Porém, após o desmame, os cordeiros de parto duplo apresentaram maior ganho de peso (98 e $117 \mathrm{~g} / \mathrm{dia})(\mathrm{P} \leq 0,05)$ e uma superioridade $(\mathrm{P} \leq 0,001)$ em quilogramas de cordeiros desmamados $(26,80$ e $49,61 \mathrm{~kg})$ ou embarcados $(33,91$ e $64,69 \mathrm{~kg})$ por ovelha parida. Independentemente do sexo e do tipo de parto, todos os cordeiros superaram as exigências de peso e condição corporal mínima requerida para comercialização de cordeiros pesados no Uruguai, na metade do tempo que em média ocorre com os genótipos tradicionais.
\end{abstract}

Palavras-chave: cordeiros pesados, cruzamento, gêmeos, produção de carne

\begin{abstract}
This study aimed to evaluate the effect of ram genotype (Dorper or Southdown), sex and birth type on the growth characteristics of 268 lambs from Australian Merino ewes. Until weaning all animals were kept on red clover pasture (Trifolium pretense), chicory (Cychorium intibus), white clover (Trifolium repens), fescue (Festuca arundinacea) and ryegrass (Lolium multiflorum), with a stocking rate of 10 sheep and their lambs/ha. From weaning (29.95 $\pm 4.94 \mathrm{~kg}$ of body weight and $109.45 \pm 7.41$ day-old), the lambs remained on the same pasture which was used during lactation, being subsequently finished on a soybean culture (Glycine max), with a stocking rate of 30 lambs/ha. No effect of ram genotype (P>0.05) was verified on any of the variables. On the other hand, the males (criptorquidas) presented superior weight gain from birth to weaning and from weaning until shipment of lambs for slaughter, (241 and 212g/day; 121 and 94g/day, for males and females, respectively; $P \leq 0.001)$, which provided a body weight at
\end{abstract}

Recebido em 27 de maio de 2014

Aceito em 28 de setembro de 2015

E-mail: tano@fagro.edu.uy 
Avaliação da progênie...

shipment $14.9 \%$ higher than the female $(40.89$ and $35.59 \mathrm{~kg}$, for males and females, respectively; $P \leq 0.001)$. Lambs originated from simple delivery presented higher $(P \leq 0.001)$ body weight and body measures at birth, and a higher growth rate until weaning (239 and 214g/day, simple and double delivery, respectively; $P \leq 0.001)$. Therefore, after weaning, the lambs originated from double delivery presented higher weight gain $(98$ and $117 \mathrm{~g} /$ day) $(P \leq 0.05)$ and were superior $(P \leq 0.001)$ in kilograms of weaned lambs (26.80 and $49.61 \mathrm{~kg}$ ) or shipped (33.91 and $64.69 \mathrm{~kg})$ per parity ewes. Regardless of the sex and birth type, all lambs overcame the weight and body conditions required for commercialization of heavy lambs in Uruguai in half the average time of traditional genotypes.

Keywords: heavy lambs, cross-breed, twins, meat production

\section{INTRODUÇÃO}

A produção de carne ovina no Uruguai é uma atividade que vem aumentando gradativamente nos últimos anos, principalmente em relação à produção de carne de cordeiros com vistas à colocação desse produto no mercado consumidor externo. Nesse contexto, faz-se necessário contemplar as exigências dos consumidores, relacionadas com a produção de carcaças sem excesso de gordura e com maior quantidade de carne de cordeiros pesados, que proporcionem um alto rendimento dos cortes comerciais e uma melhor apresentação (Garibotto et al., 2003a). A indústria exportadora de carne que atua no Uruguai demanda animais que cresçam rápido (para poder comercializá-los no primeiro semestre do ano, quando há menos cordeiros disponíveis), que sejam pesados, mais homogêneos e proporcionem, nas suas carcaças, cortes mais pesados e magros.

Contudo, a produção de carne ovina uruguaia é baseada, fundamentalmente, em raças especializadas na produção de lã ou, no máximo, em duplo propósito (principalmente Corriedale), as quais produzem carcaças que, na média, não ultrapassam $16,5 \mathrm{~kg}$, de pobre conformação, sem padronização e com excesso de gordura (Bianchi, 2013). Ainda há o agravante de que esse cordeiro, produto da má alimentação que recebe - além do genótipo não especializado para a produção de carne - permanece no campo de 10 a 11 meses antes de ser comercializado, concentrando-se sua venda no período de maior oferta do país, que coincide com o pico de produção de pasto do campo natural no final da primavera.

Nesse sentido, buscando uma melhoria dos aspectos quantitativos e qualitativos da carcaça dos animais, a introdução de raças especializadas para produção de carne para serem utilizadas em cruzamentos terminais com matrizes da base laneira ou mistas é uma ferramenta tecnológica que vem sendo cada vez mais empregada. Algumas iniciativas bem-sucedidas nesse sentido foram realizadas de forma experimental, validadas comercialmente, e podem ser consultadas em Bianchi e Garibotto (2007).

Entre as raças de corte que têm sido utilizadas em cruzamentos, encontra-se a Southdown, a qual produz cordeiros que apresentam rápido crescimento e com terminação precoce (Bianchi et al., 2000), aspecto esse importante quando se objetiva comercializar os animais entre 36 e $38 \mathrm{~kg}$ de peso vivo. No país existem antecedentes bem-sucedidos do cruzamento dessa raça com ovelhas Merino Australiano (Bianchi e Garibotto, 2007). Internacionalmente já se encontram, há bastante tempo, antecedentes do uso da raça Southdown (Kirton et al., 1995; Fogarty et al., 2000).

Mais recentemente foi introduzida no Uruguai a raça Dorper, animal com origem da África do Sul como produto do cruzamento de carneiros Dorset $\mathrm{x}$ ovelhas Black Head Persian (Milne, 2000), com muito boa reputação carniceira no exterior (Schoeman, 2000; Cloete et al., 2007), porém, sem antecedentes experimentais no pais, particularmente estudos que comparem o desempenho produtivo de cordeiros filhos de carneiros das raças Southdown ou Dorper, quando esses são utilizados em cruzamentos terminais com ovelhas Merino Australiano.

$\mathrm{O}$ uso de raças de corte (em cruzamentos ou puras) contribui para a melhoria da produção de carne, no entanto pode trazer consigo inconvenientes na parição, devido à hipertrofia fetal e à incidência de distocia. $\mathrm{O}$ peso ao nascer é o principal fator que determina a sobrevivência do cordeiro, e a duração do parto é a principal causa que explica a incidência de dificuldades ao 
parto (Dutra et al., 2007; Dutra e Banchero, 2011). Assim, tem-se observado que algumas medidas corporais (diâmetro do pescoço, comprimento do corpo e da perna) exercem influência na duração do parto e na ocorrência de distocia (Dutra et al., 2007). Em cordeiros gêmeos, a duração do parto, em geral, é menor e, apesar do menor peso ao nascimento, eles compensam a maior mortalidade neonatal em relação aos oriundos de parto simples, com maior quantidade de carne ao desmame e/ou na comercialização. Para isso, uma boa alimentação durante a lactação, aproveitando ao máximo a produção de pasto de qualidade na primavera e o leite da ovelha, enquanto o cordeiro se encontra ao pé da mãe, é importante para alcançar pesos ao desmame próximos ao objetivo de comercialização, particularmente em sistemas extensivos.

O objetivo do presente trabalho foi avaliar o efeito do genótipo do carneiro (Dorper ou Southdown cruzado com ovelhas Merino Australiano), do sexo e do tipo de parto sobre características corporais ao nascimento, o peso vivo e a velocidade de crescimento dos cordeiros até o desmame e pós-desmame, bem como os quilogramas de cordeiro produzidos por ovelha parida.

\section{MATERIAL E MÉTODOS}

O trabalho foi desenvolvido nas instalações da Estação Experimental "Dr. Mario A. Cassinoni”, da Faculdade de Agronomia em Paysandú, Uruguai $\left(32,5^{\circ}\right.$ de latitude sul e $58,0^{\circ}$ de longitude oeste), no período de 06 de agosto de 2013 a 08 de março de 2014.

Foram utilizados 268 cordeiros, produtos da inseminação artificial de ovelhas Merino Australiano com carneiro da raça Dorper ou Southdown, assim distribuídos: 199 1/2 Dorper x $1 / 2$ Merino Australiano (97 machos e 102 fêmeas; 128 de parto simples e 71 de parto duplo) e $691 / 2$ Southdown x 1/2 Merino Australiano (32 machos e 37 fêmeas; 54 de parto simples e 15 de parto duplo). Os cordeiros nasceram entre 06 de agosto e 16 de setembro de 2013.

Do nascimento ao desmame, os cordeiros permaneceram ao pé da mãe sob mesma condição de pastejo (pastagem cultivada de trevo-vermelho (Trifolium pratense), chicória
(Cychorium intibus), trevo-branco (Trifolium repens), festuca (Festuca arundinacea) e azevém (Lolium multiflorum), em uma carga animal de 10 ovelhas com seus cordeiros/ha.

Todos os cordeiros foram desmamados no mesmo dia, com idade média de 109,45 $\pm 7,41$ dias, ocasião em que foram pesados individualmente. A partir do desmame, os animais continuaram sob a mesma condição de pastejo pré-desmame. Posteriormente, foram terminados em um cultivo de soja (Glycine max), com carga animal de 30 cordeiros/ha.

Durante a parição, efetuavam-se duas recorridas diárias (às oito e às15 horas) para identificar os cordeiros. Quando alguma ovelha se encontrava parida, eram registrados data de nascimento, peso do cordeiro, sexo, tipo de parto e, eventualmente, distocia e morte. Adicionalmente, com auxílio de fita métrica, realizavam-se nos cordeiros as seguintes medições corporais: comprimento corporal (ao nível das cruzes do animal, final da espinha escapular, até a primeira vértebra coccígea), comprimento da perna dianteira (comprimento do úmero) e diâmetro do pescoço (ao nível da áxis, distância entre suas apófises transversas).

Os cordeiros foram assinalados com idade média de $13,78 \pm 4,76$ dias, ocasião em que foi realizado nos machos o procedimento de criptorquidismo induzido (Hudson et al., 1968), e não ocorreu o descole de nenhum dos animais. Nesse momento, os animais eram pesados individualmente.

O período pós-desmame teve a duração de 92 dias, quando, então, os cordeiros com idade média de 201,45 $\pm 7,41$ dias foram comercializados para serem abatidos. Nessa fase, para um melhor acompanhamento do desempenho, foram realizadas pesagens periódicas dos animais, a cada 14 dias. No momento anterior ao embarque, os cordeiros foram pesados e avaliados quanto ao grau de terminação, utilizando-se da escala de escore de condição corporal de 6 pontos (0-5) (Russel et al., 1969).

Para calcular os dados de quilogramas de cordeiro desmamado ou embarcado por ovelha parida, foram utilizados os dados de 205 ovelhas, incluindo-se no cálculo aqueles animais em que 
ocorreu mortalidade de cordeiros em ambas as fases de avaliação.

O delineamento experimental utilizado foi o inteiramente ao acaso, com número desigual de repetições por tratamento. Foi testado o efeito da raça do reprodutor, do sexo dos cordeiros, do tipo de parto e das possíveis interações (reprodutor x sexo, reprodutor x tipo de parto, sexo $\mathrm{x}$ tipo de parto e reprodutor $\mathrm{x}$ sexo $\mathrm{x}$ tipo de parto), por meio de análise de variância e teste $F$. Para os pesos vivos e ganhos de peso, utilizou-se a idade dos cordeiros como covariável. As médias foram comparadas pelo teste $t$ de Student adotando-se o nível de $5 \%$ de significância. Também foi calculado o coeficiente de correlação entre o peso ao nascimento, as medidas corporais dos cordeiros ao nascimento e a ocorrência de partos distócicos, sendo verificada a probabilidade de a correlação ser significativa a $5 \%$ pelo teste t. Para realização das análises, foi utilizado o pacote estatístico SAS, versão 9.1 (SAS, 2004).

\section{RESULTADOS E DISCUSSÃO}

Para as variáveis avaliadas ao nascimento, somente o tipo de parto mostrou efeito significativo $(\mathrm{P} \leq 0,001 ;$ Tab. 1). Não foram encontrados na literatura trabalhos anteriores que compararam o desempenho dos genótipos avaliados no presente trabalho. No entanto, de acordo com os resultados obtidos, é possível sugerir que, nas condições em que foi realizado esse experimento, as variáveis avaliadas em relação ao nascimento dos cordeiros dependeram do efeito materno.

Tabela 1. Valores médios e erro-padrão para o efeito da raça do reprodutor, do tipo de parto e do sexo sobre o peso vivo e algumas medidas corporais dos cordeiros ao nascimento e a ocorrência de partos distócicos

\begin{tabular}{cccccc}
\hline & $\begin{array}{c}\text { PVN } \\
(\mathrm{kg})\end{array}$ & $\begin{array}{c}\text { COMPCO } \\
(\mathrm{cm})\end{array}$ & $\begin{array}{c}\text { COMPPE } \\
(\mathrm{cm})\end{array}$ & $\begin{array}{c}\text { DIAMPES } \\
(\mathrm{cm})\end{array}$ & $\begin{array}{c}\text { DISTOCIA } \\
(\%)\end{array}$ \\
\hline Reprodutor & Ns & Ns & Ns & Ns & Ns \\
Dorper & $4,22 \pm 0,05$ & $29,35 \pm 0,16$ & $12,02 \pm 0,01$ & $9,76 \pm 0,01$ & $4,29 \pm 0,02$ \\
Southdown & $4,40 \pm 0,11$ & $29,69 \pm 0,33$ & $12,00 \pm 0,18$ & $9,44 \pm 0,16$ & $5,55 \pm 0,04$ \\
\hline Sexo & Ns & Ns & Ns & Ns & Ns \\
Macho & $4,42 \pm 0,09$ & $29,35 \pm 0,29$ & $11,95 \pm 0,16$ & $9,60 \pm 0,14$ & $3,93 \pm 0,03$ \\
Fêmea & $4,20 \pm 0,08$ & $29,68 \pm 0,23$ & $12,06 \pm 0,13$ & $9,59 \pm 0,11$ & $5,91 \pm 0,02$ \\
\hline Tipo de parto & $* * *$ & $* * *$ & $* * *$ & $* * *$ & $* * *$ \\
Simples & $4,93 \pm 0,06$ & $30,96 \pm 0.18$ & $12,52 \pm 0,01$ & $10,06 \pm 0,01$ & $9,11 \pm 0,02$ \\
Duplo & $3,70 \pm 0,11$ & $28,07 \pm 0,33$ & $11,49 \pm 0,18$ & $9,13 \pm 0,16$ & $0,73 \pm 0,03$ \\
\hline
\end{tabular}

Ns: $(\mathrm{P}>0,05) ; * * *:(\mathrm{P} \leq 0,001)$.

PVN: peso vivo ao nascimento; COMPCO: comprimento corporal; COMPPE: comprimento da perna; DIAMPES: diâmetro do pescoço; DISTOCIA: partos distócicos.

A ausência do efeito do sexo sobre o peso ao nascimento dos cordeiros, embora em contradição com outros experimentos (Miranda e McManus, 2000; Fernandes et al., 2001; Quesada et al., 2002), está de acordo com Silveira et al. (1992) e Rocha et al. (2009), e poderia, de certa forma, estar relacionada à boa alimentação que receberam suas mães nos momentos em que se define o peso ao nascimento: indiretamente pelo desenvolvimento placentário na metade da gestação e diretamente nos últimos 30 dias antes do parto, quando ocorre o máximo crescimento relativo do feto (Silveira et al., 1992). Da mesma forma, as medidas corporais dos cordeiros ao nascimento não foram influenciadas pelo sexo dos cordeiros, o que é explicado pela similaridade do peso ao nascimento, uma vez que essas variáveis estão altamente correlacionadas $(\mathrm{P} \leq 0,001)$ (Tab. 2). Por outro lado, o peso vivo ao nascimento dos cordeiros oriundos de parto simples foi $33,3 \%$ superior $(\mathrm{P} \leq 0,001)$ em relação aos de parto duplo, o que era esperado e está de acordo com o observado por Dwyer et al. (2005), Gardner et al. (2007), Abbasi et al. (2012), Castro et al. (2012) e Koritiaki et al. (2012). A presença de mais de um feto no ambiente uterino promove entre estes uma disputa por espaço e nutrientes, o que explica a superioridade de peso verificada nos animais oriundos de parto simples. Como uma consequência do maior peso ao nascimento, os cordeiros de parto simples também 
apresentaram superioridade $\quad(\mathrm{P} \leq 0,001) \quad \mathrm{em}$ relação às medidas corporais avaliadas.

Já em relação à ocorrência de distocia, verificouse efeito significativo $(\mathrm{P} \leq 0,001)$ apenas para o tipo de parto, e a maioria dos partos distócicos ocorreu nos cordeiros de parto simples $(92,6 \%$ do total verificado), aspecto esse explicado pela correlação significativa (Tab. 2) dessa variável com peso vivo $(\mathrm{P} \leq 0,05)$ e comprimento corporal $(\mathrm{P} \leq 0,001)$ dos cordeiros ao nascimento. O total de partos distócicos verificado foi de 9,84\%, aspecto esse explicado pelo pequeno tamanho corporal e pela proporção pélvica de ovelhas Merino Australiano quando cruzadas com carneiros de raça de corte. Esse resultado está de acordo com Garibotto et al. (2003b), que, ao avaliarem aspectos reprodutivos em rebanhos comerciais, verificaram, em uma das propriedades rurais avaliadas, $11,1 \%$ de partos distócicos no cruzamento de carneiros Southdown com ovelhas Merino Australiano. No entanto, o aspecto de que haja problemas na parição, não significa que os cordeiros irão morrer, se houver atenção adequada quando esse tipo de problema ocorrer, pois o atendimento durante o parto é a principal forma de diminuir a mortalidade de cordeiros por distocias (Nóbrega Jr. et al., 2005). Os resultados das correlações apresentadas na Tab. 2 indicam que o peso vivo ao nascimento e o comprimento corporal dos cordeiros foram as variáveis significativamente correlacionadas com a ocorrência de distocia, o que está de acordo com Dutra e Banchero (2011).

Tabela 2. Correlação entre a ocorrência de partos distócicos, peso vivo ao nascer e medidas corporais ao nascimento dos cordeiros

\begin{tabular}{ccccc}
\hline & DISTOCIA & PVN & COMPCO & COMPPE \\
\hline PVN & 0,16 & & & \\
COMPCO & $*$ & & & \\
& 0,21 & 0,63 & & \\
LPERNA & $* * *$ & $(16)$ & & \\
& $(16)$ & 0,44 & 0,50 & \\
DIAMPES & 0,014 & $* * *$ & $* * *$ & \\
& Ns & $(16)$ & $(16)$ & \\
& $(16)$ & 0,40 & 0,44 & 0,58 \\
& 0,032 & $* * *$ & $* * *$ & $(16 *)$ \\
\hline
\end{tabular}

Ns: $(\mathrm{P}>0,05) ; *$ : $(\mathrm{P} \leq 0,05) ; * * *$ : $(\mathrm{P} \leq 0,001)$. ( ) Número de observações.

DISTOCIA: partos distócicos; PVN: peso vivo ao nascimento; COMPCO: comprimento corporal; COMPPE: comprimento da perna; DIAMPES: diâmetro do pescoço.

Para as variáveis de crescimento dos cordeiros, somente houve interação entre genótipo do carneiro e tipo de parto (Tab. 3) para o ganho de peso do nascimento à assinalação, e os cordeiros filhos de carneiro Dorper e de parto simples apresentaram ganho de peso superior em relação aos de parto duplo, porém não diferindo dos filhos de Southdown, que também não diferiram entre si. Contudo, a velocidade de crescimento quando ao pé da mãe e o peso ao desmame dos cordeiros não foram influenciados $(\mathrm{P}>0,05)$ pelo genótipo do carneiro.

Verificou-se também que o ganho de peso diário da assinalação ao desmame e do nascimento ao desmame (Tab. 3) foi superior $(\mathrm{P} \leq 0,001)$ nos machos em relação às fêmeas, resultado explicado pelo dimorfismo sexual e pela influência hormonal masculina comum a todas as espécies mamíferas na fase de desenvolvimento e na idade adulta. Esse resultado sugere que a prática do criptorquidismo induzido em cruzamentos terminais constitui uma solução prática aos problemas reprodutivos devido à manutenção de cordeiros não castrados no rebanho e, da mesma forma, favorecendo-se do maior ritmo de crescimento dos animais submetidos a esse procedimento em relação às fêmeas. 
Avaliação da progênie...

Tabela 3. Valores médios e erro-padrão para o efeito da raça do reprodutor, do tipo de parto e do sexo sobre o peso vivo e a velocidade de crescimento dos cordeiros do nascimento ao desmame

\begin{tabular}{cccccc}
\hline & $\begin{array}{c}\text { PVSIN } \\
(\mathrm{kg})\end{array}$ & $\begin{array}{c}\text { PVDSM } \\
(\mathrm{kg})\end{array}$ & $\begin{array}{c}\text { GMDNASI } \\
(\mathrm{g} / \mathrm{dia})\end{array}$ & $\begin{array}{c}\text { GMDSID } \\
(\mathrm{g} / \mathrm{dia})\end{array}$ & $\begin{array}{c}\text { GMDNAD } \\
(\mathrm{g} / \mathrm{dia})\end{array}$ \\
\hline Reprodutor & $\mathrm{Ns}$ & $\mathrm{Ns}$ & $\mathrm{Ns}$ & $\mathrm{Ns}$ & $\mathrm{Ns}$ \\
Dorper & $9,36 \pm 0,10$ & $29,44 \pm 0,40$ & $375 \pm 0,01$ & $211 \pm 0,01$ & $230 \pm 0,01$ \\
Southdown & $9,54 \pm 0,21$ & $28,80 \pm 0,66$ & $392 \pm 0,01$ & $209 \pm 0,01$ & $224 \pm 0,01$ \\
\hline Sexo & $* *$ & $* * *$ & $\mathrm{Ns}$ & $* * *$ & $* * *$ \\
Macho & $9,77 \pm 0,18$ & $30,87 \pm 0,60$ & $397 \pm 0,01$ & $225 \pm 0,01$ & $241 \pm 0,01$ \\
Fêmea & $9,13 \pm 0,14$ & $27,36 \pm 0,48$ & $370 \pm 0,01$ & $194 \pm 0,01$ & $212 \pm 0,01$ \\
\hline Tipo de parto & $* * *$ & $* * *$ & $* * *$ & $*$ & $* * *$ \\
Simples & $10,41 \pm 0,11$ & $31,07 \pm 0,38$ & $412 \pm 0,01$ & $217 \pm 0,01$ & $239 \pm 0,01$ \\
Duplo & $8,49 \pm 0,20$ & $27,16 \pm 0,66$ & $355 \pm 0,01$ & $202 \pm 0,01$ & $214 \pm 0,01$ \\
\hline Rep*parto & & & & & \\
Dorper simples & & & $420 \pm 0,01 \mathrm{a}$ & & \\
Dorper duplo & & $330 \pm 0,01 \mathrm{~b}$ & & \\
South. Simples & & $403 \pm 0,01 \mathrm{a}$ & & \\
South. Duplo & & $380 \pm 0,02 \mathrm{ab}$ & & \\
\hline
\end{tabular}

Ns: $(\mathrm{P}>0,05) ; *:(\mathrm{P} \leq 0,05) ; * *:(\mathrm{P} \leq 0,01) ; * * *:(\mathrm{P} \leq 0,001)$.

PVSIN: peso vivo na assinalação; PVDSM: peso vivo ao desmame; GMDNASI: ganho de peso médio diário do nascimento à assinalação; GMDSID: ganho de peso médio diário da assinalação ao desmame; GMDNAD: ganho de peso médio diário do nascimento ao desmame.

Com relação ao efeito do tipo de parto, observase que os cordeiros de parto simples apresentaram ganho de peso superior $(\mathrm{P} \leq 0,001)$ do nascimento até o desmame, determinando maior peso vivo desses no desmame. Esse resultado se explica pela maior capacidade de extração de leite do úbere materno conforme o cordeiro é mais pesado e também porque, embora as ovelhas que amamentam dois cordeiros produzam mais leite em relação às que amamentam somente um, a quantidade de leite ingerida por um cordeiro de parto duplo é menor que aquela ingerida por um cordeiro de parto simples (Mazzitelli, 1983). Corroborando esse resultado, encontram-se os trabalhos conduzidos por Roda et al. (1990), Castro et al. (2012) e Koritiaki et al. (2012).

Durante o período pós-desmame dos cordeiros, os ganhos de peso foram inferiores aos do período de lactação (Tab. 4), o que se explica não somente pela separação da unidade ovelha/cria e pela ausência do leite materno na dieta dos cordeiros, mas também porque os cordeiros foram desmamados com um elevado peso vivo (média de $29,95 \pm 4,94 \mathrm{~kg}$ ), próximo ao começo da deposição de tecido adiposo, que apresenta um maior gasto energético em relação ao tecido muscular.
Não foi observado efeito do genótipo do carneiro em relação às variáveis analisadas no período pós-desmame. O fato de a raça Dorper estar sendo classificada como precoce em relação à deposição de gordura (Webb e Casey, 1995), bem como a raça Southdown (Bianchi et al., 2000), serve para explicar a ausência de diferenças entre as raças quanto às características avaliadas. A precocidade ao engorde, somada a uma boa velocidade de crescimento, sobretudo no período pré-desmame, posiciona muito bem qualquer um dos dois genótipos de carneiro utilizados e sugere a necessidade de disponibilizar uma melhor alimentação nesse período, no qual os animais apresentam o maior potencial de crescimento pós-natal durante a lactação. Ambos os cruzamentos superaram as exigências atualmente vigentes no Uruguai de peso e condição corporal mínima requeridas para comercialização de cordeiros pesados (como "cordeiro pesado precoce": $>32 \mathrm{~kg}$ de peso vivo e $\geq 3,5$ de condição corporal no estabelecimento; Pereira e Coronel, 2001) e, assim, é possível afirmar que esses genótipos podem ser indicados para cruzamentos industriais objetivando a produção desse tipo de animal. 
Tabela 4. Valores médios e erro-padrão para o efeito da raça do reprodutor, do tipo de parto e do sexo sobre o peso vivo e a condição corporal dos cordeiros no embarque, o ganho de peso pós-desmame e os quilogramas de cordeiro produzidos por ovelha parida

\begin{tabular}{cccccc} 
& $\begin{array}{c}\text { PVEMB } \\
(\mathrm{kg})\end{array}$ & $\begin{array}{c}\text { ECC } \\
(0-5)\end{array}$ & $\begin{array}{c}\text { GMDDEMB } \\
(\mathrm{g} / \mathrm{dia})\end{array}$ & $\begin{array}{c}\text { kgCDOP } \\
(\mathrm{kg})\end{array}$ & $\begin{array}{c}\mathrm{KgCEOP} \\
(\mathrm{kg})\end{array}$ \\
\hline Reprodutor & $\mathrm{Ns}$ & $\mathrm{Ns}$ & $\mathrm{Ns}$ & $\mathrm{Ns}$ & $\mathrm{Ns}$ \\
Dorper & $38,31 \pm 0,46$ & $3,69 \pm 0,03$ & $103 \pm 0,004$ & $37,47 \pm 1,41$ & $47,57 \pm 1,85$ \\
Southdown & $38,17 \pm 0,76$ & $3,58 \pm 0,05$ & $113 \pm 0,006$ & $38,94 \pm 2,26$ & $51,02 \pm 2,97$ \\
\hline Sexo & $* * *$ & $\mathrm{Ns}$ & $* * *$ & ----- & ----- \\
Macho & $40,89 \pm 0,68$ & $3,63 \pm 0,05$ & $121 \pm 0,006$ & ----- & ---- \\
Fêmea & $35,59 \pm 0,56$ & $3,64 \pm 0,04$ & $94 \pm 0,005$ & ---- & ---- \\
\hline Tipo de parto & $* *$ & $\mathrm{Ns}$ & $*$ & $* * *$ & $* * *$ \\
Simples & $39,45 \pm 0,44$ & $3,65 \pm 0,03$ & $98 \pm 0,003$ & $26,80 \pm 0,97$ & $33,91 \pm 1,28$ \\
Duplo & $37,02 \pm 0,77$ & $3,62 \pm 0,05$ & $117 \pm 0,006$ & $49,61 \pm 2,47$ & $64,69 \pm 3,26$ \\
\hline
\end{tabular}

Ns: $(\mathrm{P}>0,05) ; *:(\mathrm{PV} \leq 0,05) ; * *:(\mathrm{P} \leq 0,01) ; * * *:(\mathrm{P} \leq 0,001)$.

PVEMB: peso vivo ao embarque; ECC: escore de condição corporal; GMDDEMB: ganho de peso médio diário do desmame ao embarque; kgCDOP: quilogramas de cordeiro desmamado por ovelha parida; kgCEOP: quilogramas de cordeiro embarcado por ovelha parida.

Em relação ao desempenho dos cordeiros machos e fêmeas, como consequência das diferenças de ganho de peso entre os sexos (resultado do dimorfismo sexual comentado anteriormente), verificou-se uma superioridade de $14,9 \%$ de peso ao embarque dos machos em relação às fêmeas $(5,3 \mathrm{~kg}$ de peso vivo/cordeiro), aspecto esse importante do ponto de vista econômico.

$\mathrm{Na}$ Tab. 4, também se observa que o ganho de peso diário do desmame ao embarque foi superior $(\mathrm{P} \leq 0,05)$ nos cordeiros de parto duplo, demonstrando a possibilidade de se explorar esse tipo de animal em termos de ganhos compensatórios de peso quando submetido, na fase pós-desmame, a uma condição de boa alimentação, desde que anteriormente, na fase pré-desmame, seu crescimento não tenha sido severamente restringido do ponto de vista alimentar.

Outro aspecto importante em relação à ocorrência de partos múltiplos se refere ao potencial de produção de carne desse animal, podendo-se verificar a grande superioridade $(\mathrm{P} \leq 0,001)$ quando se avaliam os quilogramas de cordeiros desmamados ou embarcados por ovelha parida (Tab. 4). A produção de carne dos cordeiros de parto duplo é quase o dobro do que a dos de parto simples, o que compensa seu menor crescimento individual, justificando dispor de boas condições de alimentação pósparto que permitam expressar boas taxas de ganho de peso diário, independentemente do tipo de parto, tal qual ocorreu no presente trabalho.

\section{CONCLUSÕES}

Carneiros das raças Dorper e Southdown podem ser recomendados para serem utilizados em cruzamentos terminais com ovelhas Merino Australiano, produzindo cordeiros precoces e pesados, de forma mais rápida e econômica, em comparação ao sistema tradicional baseado exclusivamente em raças de lã. Com boas condições de alimentação no período pós-parto, a incidência de partos duplos é chave para quase duplicar os quilogramas de carne de cordeiros comercializados.

\section{REFERÊNCIAS}

ABBASI, A.; ABDOLLAHI-ARPANAHIB. R.; MAGHSOUDIC, A. et al. Evaluation of models for estimation of genetic parameters and maternal effects for early growth traits of Iranian Baluchi sheep. Small Rumin. Res., v.104, p.6269, 2012.

BIANCHI, G. Confinamiento de corderos. In: SEMINÁRIO DE ACTUALIZACION TÉCNICA: PRODUCCIÓN DE CARNE OVINA DE CALIDAD, 2013, Treinta y Tres, UY. Anais... Treinta y Tres: INIA, 2014. p.101-112. (Serie Actividades de Difusión, 719). 
BIANCHI, G.; GARIBOTTO, G. Uso de razas carniceras en cruzamientos terminales y su impacto en la producción de carne y el resultado económico. In: BIANCHI G. (Ed.). Alternativas tecnológicas para la producción de carne ovina de calidad en sistemas pastoriles. Montevideo, Uruguay: Hemisferio Sur, 2007. p.65-106.

BIANCHI, G.; GARIBOTTO, G.; BENTANCUR, O. Relation between cold carcass weihgt and tissue depth in gr site: effect of breed and sex in pure and crossbred heavy lambs of 5 months of age. In: INTERNATIONAL CONGRESS OF MEAT SCIENCE \& TECHNOLOGY, 46., 2000, Buenos Aires. Anais... Buenos Aires: [s.n.]. 2000. p.358-359.

CASTRO, F.A.B.; RIBEIRO, E.L.A.; KORITIAKI, N.A. et al. Desempenho de cordeiros Santa Inês do nascimento ao desmame filhos de ovelhas alimentadas com diferentes níveis de energia. Sem. Ciênc. Agra., v.33, p.3379-3388, 2012.

CLOETE, J.J.E.; CLOETE, S.W.P.; OLIVIER, J.J. et al. Terminal crossbreeding of Dorper ewes to Ile de France, Merino Landsheep and SA Mutton Merino sires: ewe production and lamb performance. Small Rumin. Res., v.69, p.28-35, 2007.

DUTRA, F.; BANCHERO G. Polwarth and Texel ewe parturition duration and its association with lamb birth asphyxia. J. Anim. Sci., v.89, p.3069-3078, 2011.

DUTRA, F.; QUINTANS, G.; BANCHERO, G. Lesions in the central nervous system associated with perinatal lamb mortality. Aust. Vet. J., v.85, p.405-413, 2007.

DWYER, C.; CALVERT, S.; FARISH, M. et al. Breed, litter and parity effects on placental weight and placentome number, and consequences for the neonatal behaviour of the lamb. Theriogenology, v.63, p.1092-1110, 2005.

FERNANDES, A.A.O.; BUCHANAN, D.; SELAIVE-VILLARROEL, A.B. Avaliação de fatores ambientais no desenvolvimento corporal de cordeiros deslanados da raça Morada Nova. Rev. Bras. Zootec., v.30, p.1460-1465, 2001.

FOGARTY, N.M.; HOPKINS, D.L.; VAN DE VEN, R. Lamb production from divers genotypes 2. Carcass characteristics. Anim. Sci., v.70, p.147-156, 2000.
GARDNER, D.; BUTTERY, P.; DANIEL, Z. et al. Factors affecting birth weight in sheep: maternal environment. Reproduction, v.133. p.297-307, 2007.

GARIBOTTO, G.; BIANCHI, G.; FRANCO, J. et al. Efecto del sexo y del largo de lactancia sobre el crecimiento, características de la canal y textura de la carne de cordeiros Corriedale sacrificados a los 5 meses de edad. Agrociencia, v.7, p.19-29, 2003a.

GARIBOTTO, G.; ARBELETCHE, P.; BIANCHI, G. Producción de carne ovina de calidad: evaluación física y econômica en base al análisis de registros de nueve empresas. Rev. EEMAC, v.23, p. 41-48, 2003 b.

HUDSON, L.W.; GIMP, H.A.; WOOLFOLK, P.G. et al. Effect of induced cryptorchidism at different weights on performance and carcass traits of lambs. J. Anim. Sci., v.27. p.45-47, 1968.

KIRTON, A.H.; CARTER, A.H.; CLARKE, J.N. et al. A comparison between 15 ram breeds for export lamb production. 1. Liveweigths, body components, carcass components, carcass measurements and composition. New Zeal. Soc. Agric. Res., v.38, p.347-360, 1995.

KORITIAKI, N.A.; RIBEIRO, E.L.A.; SCERBO, D.C. et al. Fatores que afetam o desempenho de cordeiros Santa Inês puros e cruzados do nascimento ao desmame. Rev. Bras. Saúde Prod. Anim., v.13, p.258-270, 2012.

MAZZITELI, F. Algunas consideraciones sobre crecimiento de corderos. SUL Boletin Técnico, n.8, p.53-61, 1983.

MILNE, C. The history of the Dorper sheep. Small Rumin. Res., v.36, p.99-102, 2000.

MIRANDA, R. M.; McMANUS, C. Desempenho de ovinos Bergamácia na Região de Brasília. Rev. Bras. Zootec., v.29, p.1661-1666, 2000.

NÓBREGA JUNIOR, J.E.; RIET-CORREA, F.; NÓBREGA, R.S. et al. Mortalidade perinatal de cordeiros no semi-árido da Paraíba. Pesqui. Vet. Bras., v.25, p.171-178, 2005.

PEREIRA, D.Y.; CORONEL, F. Cordero precoz pesado SUL: una propuesta productiva que apunta a la calidad. SUL Lanonot., v.129, p.25$27,2001$. 
QUESADA, M.; McMANUS, C.; COUTO, F.A.A. Efeitos genéticos e fenotípicos sobre características de produção e reprodução de ovinos deslanados no Distrito Federal. Rev. Bras. Zootec., v.31, p.342-349, 2002.

ROCHA, L.P.; FRAGA, A.B.; ARAÚJO FILHO, J.T.; FIGUEIRA, R.F. Desempenho de cordeiros cruzados em Alagoas, Brasil. Arch.. Zootec., v.58, p.145-148, 2009.

RODA, D.S.; OTTO, P.A.; SANTOS, L.E. et al. Efeito do tipo de gestação (simples ou gemelar) na sobrevivência e desenvolvimento de cordeiros das raças Ideal e Corriedale. Bol. Ind. Anim., v.47, p.115-119, 1990.

RUSSEL, A.J.F.; DONEY, J.M.; GUNN, R.G. Subjective assessment of body fat in live sheep. J. Agric. Sci. Camb., v.72, p.451-454, 1969.
SCHOEMAN, S.J. A comparative assessment of Dorper sheep in different production environments and systems. Small Rumin. Res., v.36, p.137-146, 2000.

SILVEIRA, V.C.P.; LÓPEZ, J; RODRIGUES, F.E. Influência da nutrição materna e do sexo na reserva energética do cordeiro ao nascer. Rev. Bras. Zootec., v.21, p.242-249, 1992.

STATISTICAL Analisys System - SAS: user's guide, version 9.1. North Caroline: SAS Institute INC., 2004. 5135p.

WEBB, E.C.; CASEY, N.H. Genetic differences in fatty acid composition of subcutaneous adipose tissue in Dorper and SA Mutton Merino wethers at different live weights. Small Rumin. Res., v.18, p.81-88, 1995. 